\title{
Ultrafast Laser Applications in Low Dimensional Systems
}

\author{
Prem B. Bisht
}

Physics Department, IIT Madras, Chennai 600036, India

E-mail: bisht@iitm.ac.in

\begin{abstract}
Among several laser applications, nonlinear optical phenomena have emerged as a new area of research. Nonlinear optics of low dimensional systems such as quantum dots (0D), carbon nano tubes (1D) and graphene derivatives (2D) has been under intense scrutiny. In the present manuscript we present some of our research work in these topics carried out with a simple technique of z-scan. This technique is useful for the studies of novel materials and can be replicated in a laboratory that has a pulsed (ns or shorter duration) laser facility.
\end{abstract}

Keywords: Nonlinear optics, Ultrafast Laser, Z-scan.

\section{Introduction}

When high intense laser pulses interact with materials, several nonlinear phenomena can take place. These nonlinear effects depend on the peak power of the input pulse. High peak power can be achieved from a short duration pulse with high pulse energy. Pulses of ns or shorter duration are required to study such effects as the electric field (E) of the laser is of the order of the inter-atomic field. The higher order nonlinearities are invoked in a sample and the components of the induced polarisation $(\mathrm{P})$ can be written in the tensor form

$P_{i}=\varepsilon_{0}\left[\chi_{\mathrm{ij}} E_{j}+\chi_{\mathrm{ijk}}^{(2)} \mathrm{E}_{\mathrm{j}} \mathrm{E}_{\mathrm{k}}+\chi_{\mathrm{jjk}}^{(3)} \mathrm{E}_{\mathrm{j}} \mathrm{E}_{\mathrm{k}} \mathrm{E}_{1}+\cdots\right]$

Or $\widetilde{P}(t)=\widetilde{P_{1}}(t)+\widetilde{P_{2}}(t)+\widetilde{P_{3}}(t)+\cdots \cdots$

Here $\chi_{i j k_{m}}^{(n)}(n=2,3, \ldots)$ is the $n^{\text {th }}$ order susceptibility tensor of rank $n+1$. For example, the linear susceptibility, $x_{\mathrm{ij}}$ is a dimensionless quantity with 9 components as follows.

$$
\chi_{i j}=\left[\begin{array}{lll}
\chi_{11} & \chi_{12} & \chi_{13} \\
\chi_{21} & \chi_{22} & \chi_{23} \\
\chi_{31} & \chi_{32} & \chi_{33}
\end{array}\right]
$$

In eq. (1), in order to make $\widetilde{P_{2}}(t)$ to be comparable to $\tilde{P}(t)$, we can see that $\widetilde{P_{2}}(t)=\epsilon_{0}\left(\chi^{(2)} E\right) E \quad$ or $\epsilon_{0}(\Delta \chi) E$. Here $\chi^{(2)} E$ is the change of susceptibility ( ). Therefore, the $\chi^{(2)}$ now has the dimensions of inverse of E. Its units are written as $\mathrm{m} / \mathrm{V}$. Similarly, the units of $\chi^{(3)}$ are $\mathrm{m}^{2} / \mathrm{V}^{2}$. Table 1 gives the order of magnitude of various nonlinear optical susceptibilities.

Table 1. Estimates of the second and third order optical nonlinear susceptibilities

\begin{tabular}{lll}
\hline Susceptibility & $\begin{array}{l}\text { Order of } \\
\text { magnitude }\end{array}$ & Units \\
\hline$\chi$ & 1 & No units \\
$\chi^{(2)}$ & $10^{-10}$ & $\mathrm{~m} / \mathrm{v}$ \\
$\chi^{(3)}$ & $10^{-20}$ & $\mathrm{~m}^{2} / \mathrm{v}^{2}$ \\
\hline
\end{tabular}

The net refractive index (n) in terms of nonlinear refractive index $\left(n_{2}\right)$ and irradiance (I) is written as 
$n=n_{0}+n_{2} I$

Here $n_{0}$ is the refractive index of the medium (at low electric fields). This is also known as optical Kerr effect (OKE) due to its similarity with the DC Kerr effect. Eq. (2) has profound applications in nonlinear optics and laser technology. In the following sections, some of the applications are described.

For example, one of the third order nonlinear optical process is the two photon absorption process. In two photon absorption process, a molecule makes the transition from the ground state to the excited state by simultaneously absorbing two photons of frequency $v_{1}$. It is followed by the emission of a photon of frequency $2 v_{1}$ (Fig. 1). Two photon absorption (2PA) has several technological applications such as frequency upconversion lasing, optical limiting, multiphoton imaging, and multiphoton associated medical and biological applications.

Figure 1: Energy level diagram for two-photon absorption process. The dashed line is the virtual level.

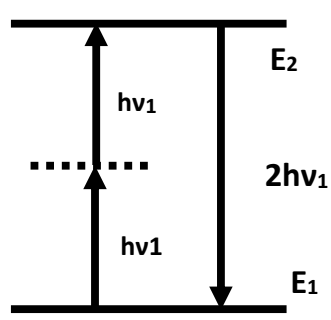

\section{Materials}

The charge transfer (CT) interaction in silver nanoparticle (AgNP) decorated graphene (fG) as well as single walled carbon naotubes (SWCNT) with laser dye rhodamine 6G (R6G) has been studied in this work.

It is known that a single layer of graphene absorbs about $2.3 \%$ of light incident on it, and the multilayered graphene absorbs in the same proportion Due to its zero band gap nature, absorption of electromagnetic radiation of any wavelength is possible Silver nanoparticles (AgNPs) have been found be useful in various applications such as plasmon-controlled fluorescence, multiphoton plasmon-resonance microscopy, femtosecond filamentation and super continuum generation Importantly, these features differ from those of the bulk silver and actually depend on the size and shape of the AgNPs.

\section{Experimental: Z-scan technique}

The nonlinear optical measurements were done by the open aperture (OA) Z-scan setup (Fig. 2). Here, the fundamental (1064 nm) and the second harmonic of the Nd: YAG laser $(532 \mathrm{~nm}, 30 \mathrm{ps}$ and $10 \mathrm{~Hz})$ were used as the pump wavelengths. The sample was irradiated and scanned in the focal plane of the $\quad 5 \quad \mathrm{~cm}$ double

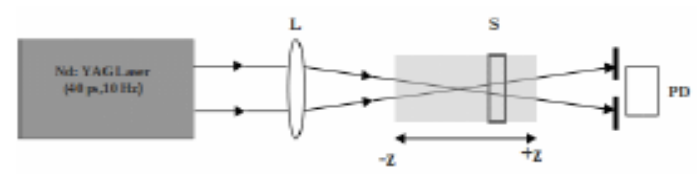

Figure 2: The experimental setup for Z-scan, L - lens, S - sample, PD - photodiode

convex lens. All the transmitted light was collected by a large aperture double convex lens and detected by the PD 400 photo diode. By measuring the transmitted intensity, this method enabled the researchers to study nonlinear absorption in the material and clearly identify the saturable absorption (SA) or reverse saturable absorption (RSA) threshold at the pump wavelength.

\section{Theory}

TPA is a nonlinear absorption process. The transmitted signal in the OA Z-scan setup for 
Gaussian beam under low irradiance limit is given by [5]

$$
T(z)=1-\left(\beta I_{0} L_{\text {eff }} / 2\left(x^{2}+1\right)\right)
$$

\section{(3)}

Where, $\beta$ is the nonlinear absorption coefficient, $I_{0}$ is the input irradiance at the focus, $L_{\text {eff }}(=1-\exp (-\alpha L) / L)$ is the effective sample length in which $\alpha$ is linear absorption coefficient and $\mathrm{L}$ is sample length. $x=z / z 0, z 0$ is Rayleigh range. The imaginary part of the third order nonlinear susceptibility $\chi^{(3)}$ is given by

$$
\chi_{i m}^{(3)}=\frac{1}{3 \pi} n_{0}^{2} \epsilon_{0} c \lambda \beta
$$

Here, $n_{0}$ is the linear absorption coefficient, $\varepsilon_{0}$ is the dielectric permittivity of free space, $c$ is the light velocity, and $\lambda$ is the excitation wavelength.

\section{Results and discussion}

\section{Graphene-AgNP system}

When graphene (a 2D material) and AgNPs are in physical contact, electron transfer takes place from fG to the AgNPs to achieve a common Fermi level. Under illumination, electrons from valence band (VB) are excited to the conduction band $(\mathrm{CB})$ as shown in fig. 3

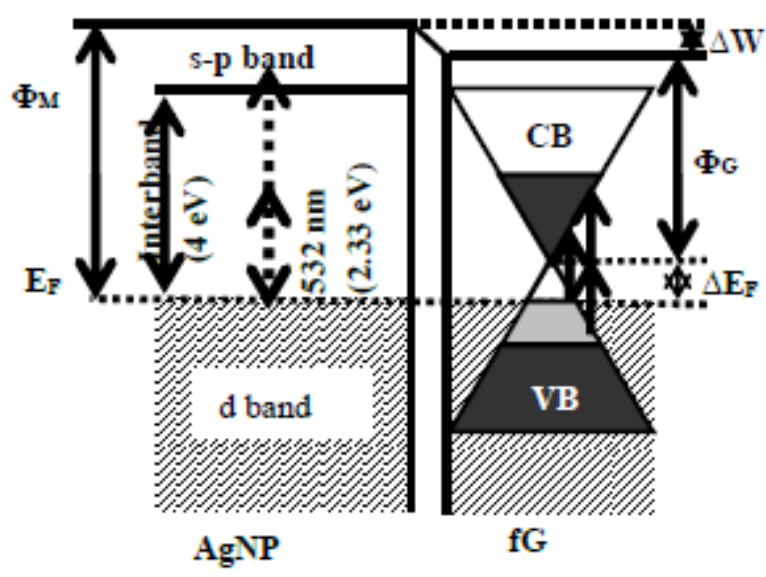

Figure 3: The energy diagram of the metalgraphene interface and resulting Fermi level with respect to the Dirac point. Fermi level $\left(E_{F}\right)$ shifts due to the electronic interaction. The work functions of the $\mathrm{Ag}\left(\varphi_{\mathrm{M}}=5.0 \mathrm{eV}\right)$ and $\mathrm{fG}\left(\varphi_{\mathrm{G}}=4.5 \mathrm{eV}\right)$ are also indicated. $\Delta \mathrm{W}$ is caused by the charge built up at the interface.

Due to considerably larger density of states in the metal, the excited electrons have higher probability of moving to the metal states across the surface than to the graphene valence bands. The excited carriers from graphene move to metal states before returning to the VB. Besides providing longer lifetimes, the transferred electrons eventually return to graphene, but the time scale is much larger than 40 ps time scale of the laser pulse. Since the carriers are excited faster than their relaxation time to the original state, the bleaching of the ground state takes place, resulting in the behaviour of saturable absorption (SA). The observed nonlinearity in $\mathrm{AgNP} / \mathrm{fG}$ at low intensity at $1064 \mathrm{~nm}$ and 532 $\mathrm{nm}$ can be attributed to the charge transfer from the graphene to silver nanoparticles [Kalanoor et. al.]. As the intensity is increased, the TPA dominates over the SA that results in nonlinear transmission as shown in fig 4.

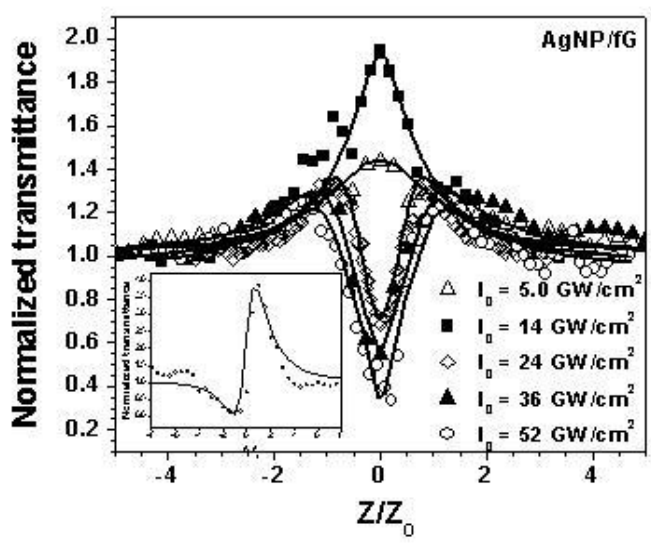


Figure 4: OA Z-scan profile of the $\mathrm{AgNP} / \mathrm{fG}$ at $1064 \mathrm{~nm}$ at different pump energies with constant concentration $1 \mathrm{mg} / \mathrm{ml}$. Inset shows the CA Z-scan profile at an intensity of 45 $\mathrm{GW} / \mathrm{cm}^{2}$.

\section{R6G-SWCNT system}

The single walled carbon nano tubes (SWCNTs ) are 1D system. The absorption spectra of R6G-SWCNT mixtures show evidence of charge transfer complex. This is due to the electrostatic interaction and $\pi-\pi$ stacking between R6G and SWCNT. The work function of R6G and SWCNT are -1.76 $\mathrm{eV}$ and $-5.05 \mathrm{eV}$, respectively. It indicates the electron transfer is favorable from SWCNT to R6G. On increasing [SWCNT], the fluorescence intensity of R6G is suppressed gradually without appearance of any new bands indicating the non-fluorescent nature of the ground state complex.

The open aperture Z-scan profiles of R6G with varying concentrations of SWCNT are shown in fig. 5. R6G exhibits the effect of saturable absorption alone. On increasing the SWCNT concentration the strength of the SA behaviour decreases drastically. The trend continued for high concentration SWCNT also. The nonlinear optical parameters of R6G-SWCNT mixture were deduced by using equation (3) and (4). $\beta$ values of $\sim 10^{-9} \mathrm{~m} / \mathrm{W}$ were obtained.

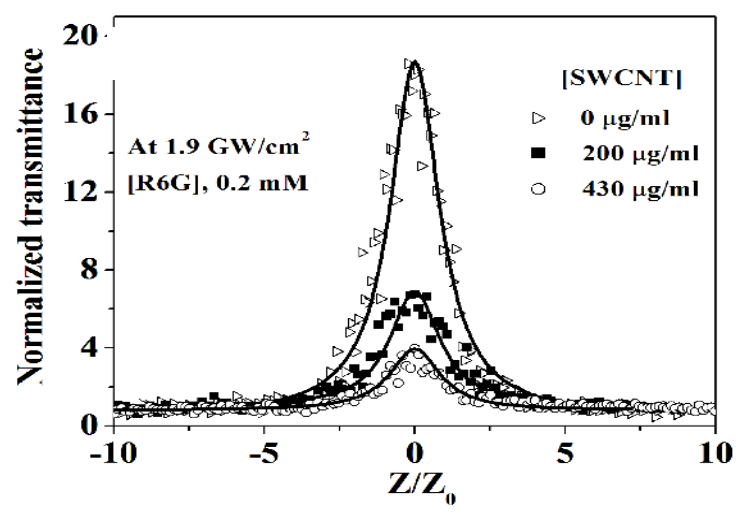

Figure 5: OA Z-scan profiles of R6G with varying concentrations of SWCNT.

\section{Conclusions}

This paper gives two examples of a simple nonlinear optical technique of z-scan that has been used to study the effect of charge transfer in novel 1D and 2D nanomaterials (with organic dyes and silver nanoparticles, respectively).

\section{Acknowledgements}

We thank Dr. Sudhakara Reddy and Dr. Basanth Kalanoor for carrying out some of the measurements. We thank Dr. Ramaprabhu for giving graphene for the experiments.

\section{References}

Kalanoor, B. S., Bisht, P. B., Ali, A. S., Baby, T. T. \& Ramaprabhu, S. Optical nonlinearity of silver-decorated graphene. J. Opt. Soc. Am. B Opt. Phys. 29, (2012).

Bongu, S. R. \& Bisht, P. B. Nonlinear optical properties of single walled carbon nanotubeslaser dye system under picosecond pumping. in Proc. Photonics (2014). M4A.34. 